\title{
Subject Index Vol. 14, 1994
}

Abdominal surgery 6 Abbot Bertario 477 Acid-base imbalance 201 Acute focal bacterial nephritis 72 $-$

Adhesion 37 renal failure $6,72,148$

Adult T cell leukemia/lymphoma 226

Adynamicbone 19

Age of Enlightenment 461

Agnogenic myeloid metaplasia 223

Alberto de' Zancari 313

Albuminuria 365,371

Alchemy 295

Alveolar hemorrhage 47

Ambrosian codex 317

Ambulatory nuclear vest 173

Amiloride 127

Anatomopathology 290

Anatomy 270

Ancient medicine 295

Anemia 95

Angiotensin-converting enzyme inhibitors

60 Anonymous medical excerpts 488 Anti-CD4 antibody 136 Antimyeloperoxidase antibodies 99

Antineutrophil cytoplasmic autoantibodies

99 Antiproteinase 3 antibodies 99 Aquatic animals 458 Arabic medicine 270 Aristotle 302

Armanni-Ebstein lesion 448 Arteriolar nephrosclerosis 90 Arteriovenous fistula 142 Asia 295

Atomic force microscope 197 Atomism 337 Autosomal dominant polycystic kidney

disease 236 Avicenna 270

Bacteremia 9

Bacteria 37

Bartter's syndrome 127

Benedictine monasteries 259

Berger's disease 64

Beta-blockers 60

Bible 279

Bizzozero 330

Blackall 371

Bladder lesions 494

stones 432 Blood clotting 330

pressure 81

- measurement 436

Bloody urine 494 
Body homeostasis 307

Bologna 313

Bone markers 106

Bostock 365

Botanical medicines 377

Botany 259

Bright's disease 436,443

Calcitriol 106 Calcium acetate 192

carbonate 192

channel blockers 60 Caseous lesion 448 Cassiodorus 477 Cat-eye syndrome 76 Catheters 37

Cefuroxime axetil 169 19th Century 436

- Germany 443 al-Chain(IV) 30 a3-Chain(IV) 30 Children 157,169 China 295 Chinese 99

Chronic renal failure 81, 106, 207, 220 Cisplatin, nephrotoxicity 81 Classical medicine 259 Codex

Casinensis 69494

- $\quad 97$ from Montecassino 477

Coloboma 76

Constantinus Africanus 488 Continuous ambulatory peritoneal dialysis

55

- $\quad$ peritoneal dialysis 182

Cotugno 325

Cyclosporine A 1, 187

Daoism 295 ddYmice 136 Densitometry 19 Dextrose 37 Diabetes 233

- $\quad$ mellitus 448

Dietary salt 426

Dioscorides 259,418

Diuresis 201

Diuretics 377

Dropsy 365,371

Early medieval medicine 317 Echinocytes 197 Edema 432 Edible plants 423 Empiricism 257

Endoscopy 344

Endothelin 157

End-stage renal disease, anemia 14

Enzymuria 81

Epithelium 355

Erythropoietin 95,230

Essential hypertension 90, 436

Ethics 264

Extranodal lymphoma 148

Familial hypertension 432

Fast atom bombardment mass spectrom-

etry 162 Favus 467 Fluconazole 113

Focal and segmental glomerulosclerosis 90 Formularia 384 Fungal peritonitis 113,216

Galenic medicine 317 Guelman's syndrome 127 Glomerular basement membrane thinning 47

- $\quad$ sclerosis 207

Glomerulonephritis 64 
Goodpasture's syndrome 47

Gram-negative bacteremia 85

Haemophilus influenzae 67 Head trauma 145 Heart transplantation 1 Hematuria 47

Hemodialysis 9, 55, 85, 95, 173 Hemolytic uremic syndrome 67 Hemoptysis 47 Henle 355

Henoch-Schönlein purpura 467 Heparan sulfate proteoglycan 30 Hepatic dysfunction 72 Hildegarde

von Bingen 391 Hippocrates 264

recommendations 494 Histomorphometry 19 History of hypertension 432

- medicine 257, 282, 337

- nephrology $344,412,418,436$

- pharmacology 391,399 Human evolution 426 Hypercalcemia 192 Hyperparathyroidism 41, 106

Hyperphosphatemia 192 Hypokalemia 127,236 Hypomagnesemia 127

IgA nephropathy 64,136 Imipenem 182 Immunoelectron microscopy 30

501

Immunosuppression 121,187,226 Indole 207 Indoxyl sulfate 207 Innominate vein stenosis 142

Insulin-dependent diabetes mellitus 201 Intercellular adhesion molecule 1136 Intestinal phosphate

binder 192 Iron stores 95 Islam 270

Juvenal 477

Kaposi's sarcoma 121 Kidney 41,279

transplantation 121

-, complications 154

Laminin 30 Lithiasis 290 Lithium 145 Lithotomy 473 Lithotrity 473 Liver 41

LoopofHenle 355 Lupus nephritis 30 Lymphocele 154

Magic ritual 384 Magnesium deficiency 201 Maimonides 307 Marine animals 458 Mechanism 337

Medical education 257

use of salt 426

works of Galen 488 Medicinal herbs 259

plants 399,412,418,423 Medieval medicine 478 Medullary sponge kidney 213 Membranous nephritis 30 Mercury poisoning 371

Mesangial proliferative glomerulonephritis

223 Mesenteric infarction, nonocclusive 230 Microscopy 355 Micturition 494 Middle Ages

282,384,391 Molecular weight range, uremic

compounds 162 Monoclonal gammopathy 187 Morality 279 Mosaics 423 mRNA 41

Multidisciplinarity 361 Mycology 467 Myocardial ischemia 173

Naturalism 337 Neonates 6 Nephritis 443

Nephrogenic diabetes insipidus 145

Nephrolithiasis 313

Nephrology 264, 270, 290, 295, 307, 325,

361 Nephromegaly 148 Nephrotic syndrome 157, 223, 325 Non-Hodgkin's lymphoma 148 Northern blot 41

Osmoregulation 458

Papillary necrosis 213 Parathyroid hormone 19 Parathyroidectomy 106 Pathology 90

Peritoneal dialysis 6, 19, 113, 216, 230 Peritonitis 37, 154, 182 Pharmacology 384 Pharmacy 384

and magic 391 Phenytoin 162 Plasma exchange 67

therapy 67 Platelets 330 Plenck 377

Pliny the Elder 399,412

Pompeii 423 
Pott's disease 55

Primary aldosteronism 220,236

Procollagen type 1106

Prognosis 264

Prostacyclin 67

Protein binding 162

Proteinuria 60, 233, 325

PTH receptor 41

Pyrogenic reactions 85

Ravenna School of Medicine 317 Recombinant human erythropoietin, weekly

dosing 14 Renaissance 290 Renal anatomy 320, 344, 355, 461

biopsies 157

disease(s) 399,412,418,448

failure 1

function 76, 233, 302

injury 136

lymphoma 148

microstructure 320

osteodystrophy 19

pathology 361,371

physiology 461

structure 302

transplant 226

transplantation 72,220

tubule 320

Renin-angiotensin-aldosterone system 236 Renovascular hypertension 60 Retinopathy 233

Rhazes 270

Salerno School of Medicine 259, 477, 478,

483 Salt 426

monopolies 426

taxes 426 Schumlansky 320 Sestamibi imaging 173 Short-term treatment 169 Sickle cell disease 213 Single-dose pharmacokinetic studies, subcutaneous route 14 Skin cancer 121 Smoldering myeloma 187 Spain 290 Spironolactone 127 Splenectomy 67 ST segments 173

Staphylococcus aureus, nasal colonization 9 Stroke 432

Superior vena cava syndrome 142 Surgery 478

Surgical instruments 473 Symbolism of salt 426

Theophilus 282

Theories of urine formation 344 Transplant, recurrent disease 64 Transplantation 187 Treatment of women 483 Trichophyton schönieinii 467 Tuberculosis 55, 448 Type IV collagen 30

Unilateral upper limb, swelling 142 University of Bologna 361 Ur(a)emia 197,207,365 Urinalysis 443, 452 Urinary calculi 461

disorders 461

microscopy 452

sediment 443,452

stones 494

tract infection 169,213 Urine formation 302 
Uroscopy 282, 307, 365, 473, 478, 483

Vascular pathology 436 Vasculitis 99 Verapamil 41 Verticillium 216 Vitalism 337

Women's status 391 Works of Alcuin 488

- $\quad$ - Hippocrates and Pseudo-Hippo-

crates 488

502

Subject Index 